\title{
Transport Properties and Clustering of Color Sources in Nuclear Collisions
}

\section{Brijesh K Srivastava*}

Department of Physics and Astronomy, Purdue University, West Lafayette, IN-47907, USA

E-mail: brijesh@purdue.edu

Possible phase transition of strongly interacting matter from hadron to a quark-gluon plasma state have in the past received considerable interest. The clustering of color sources provides a framework of the partonic interactions in the initial stage of the collisions. The onset of deconfinement transition is identified by the spanning percolation cluster in $2 \mathrm{D}$ percolation.

The thermodynamical quantities, the temperature, and energy density derived from RHIC and LHC data and Color String Percolation Model (CSPM) are used to obtain the shear viscosity to entropy density ratio $(\eta / s)$. It was observed that the inverse of $(\eta / s)$ represents the trace anomaly $\Delta=(\varepsilon-3 P) / T^{4}$. Results are in agreement with the Lattice Quantum Chromo Dynamics(LQCD) simulations. Thus the clustering of color sources has a clear physical basis although it cannot be deduced directly from QCD.

7th International Conference on Physics and Astrophysics of Quark Gluon Plasma,

1-5 February, 2015

Kolkata, India

${ }^{*}$ Speaker. 

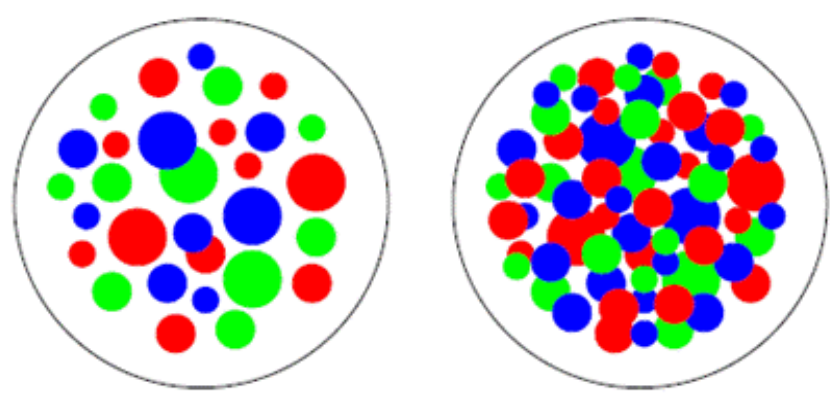

Figure 1: Partonic cluster structure in the transverse collision plane at low (left) and (right) high parton density [3].

\section{Introduction}

One of the main goal of the study of relativistic heavy ion collisions is to study the deconfined matter, known as Quark-Gluon Plasma (QGP), which is expected to form at large densities. It has been suggested that the transition from hadronic to QGP state can be treated by the percolation theory [1]. The formulation of percolation problem is concerned with elementary geometrical objects placed on a random d-dimensional lattice. The objects have a well defined connectivity radius $\lambda$, and two objects can communicate if the distance between them is less than $\lambda$. Several objects can form a cluster of communication. At certain density of the objects a infinite cluster appears which spans the entire system. This is defined by the dimensionless percolation density parameter $\xi$ [2]. Percolation theory has been applied to several areas ranging from clustering in spin system to the formation of galaxies. Figure 1 shows the parton distribution in the transverse plane of a overlapping region of low and high density partons [3].

All high energy soft multi-hadron interactions exhibit thermal patterns of abundances characterized by the same temperature, independent of the center of mass energy $[4,5]$. The hadron limiting temperatures were measured by statistical thermal analyses that fit the data with a minimum of parameters $[4,5]$. In heavy ion collisions it may be plausible that multiple parton interactions produce a thermalized system.

In this talk results are presented for the temperature, equation of state and the transport coefficient and compared with the recent LQCD simulations.

\section{Clustering of Color Sources}

Multi-particle production at high energies is currently described in terms of color strings stretched between the projectile and target. Hadronizing these strings produce the observed hadrons. At low energies only valence quarks of nucleons form strings that then hadronize. The number of strings grows with the energy and with the number of nucleons of participating nuclei. Color strings may be viewed as small discs in the transverse space filled with the color field created by colliding partons. Particles are produced by the Schwinger mechanisms [6]. With growing energy and size of the colliding nuclei the number of strings grow and start to overlap to form clusters [7, 8, 9]. At a critical density a macroscopic cluster appears that marks the percolation phase transition. This 
is termed as Color String percolation Model (CSPM) [7, 8]. The interaction between strings occurs when they overlap and the general result, due to the $\mathrm{SU}(3)$ random summation of charges, is a reduction in the multiplicity and an increase in the string tension or an increase in the average transverse momentum squared, $\left\langle p_{t}^{2}\right\rangle$. We assume that a cluster of $n$ strings that occupies an area of $S_{n}$ behaves as a single color source with a higher color field $\vec{Q}_{n}$ corresponding to the vectorial sum of the color charges of each individual string $\vec{Q}_{1}$. The resulting color field covers the area of the cluster. As $\vec{Q}_{n}=\sum_{1}^{n} \vec{Q}_{1}$, and the individual string colors may be oriented in an arbitrary manner respective to each other, the average $\overrightarrow{Q_{1 i}} \overrightarrow{Q_{1 j}}$ is zero, and $\overrightarrow{Q_{n}^{2}}=n \overrightarrow{Q_{1}^{2}}$.

Knowing the color charge $\vec{Q}_{n}$ one can obtain the multiplicity $\mu$ and the mean transverse momentum squared $\left\langle p_{t}^{2}\right\rangle$ of the particles produced by a cluster of $n$ strings [8]

$$
\mu_{n}=\sqrt{\frac{n S_{n}}{S_{1}}} \mu_{0} ; \quad\left\langle p_{t}^{2}\right\rangle=\sqrt{\frac{n S_{1}}{S_{n}}}\left\langle p_{t}^{2}\right\rangle_{1}
$$

where $\mu_{0}$ and $\left\langle p_{t}^{2}\right\rangle_{1}$ are the mean multiplicity and $\left\langle p_{t}^{2}\right\rangle$ of particles produced from a single string with a transverse area $S_{1}=\pi r_{0}^{2}$. In the thermodynamic limit, one obtains an analytic expression $[7,8]$

$$
\left\langle\frac{n S_{1}}{S_{n}}\right\rangle=\frac{\xi}{1-e^{-\xi}} \equiv \frac{1}{F(\xi)^{2}} ; \quad F(\xi)=\sqrt{\frac{1-e^{-\xi}}{\xi}}
$$

where $F(\xi)$ is the color suppression factor. $\xi=\frac{N_{S} S_{1}}{S_{N}}$ is the percolation density parameter assumed to be finite when both the number of strings $N_{S}$ and total interaction area $S_{N}$ are large. Eq. (2.1) can be written as $\mu_{n}=F(\xi) \mu_{0}$ and $\left\langle p_{t}^{2}\right\rangle_{n}=\left\langle p_{t}^{2}\right\rangle_{1} / F(\xi)$. The critical cluster which spans $S_{N}$, appears for $\xi_{c} \geq 1.2[10]$.

It is worth noting that CSPM is a saturation model similar to the Color Glass Condensate (CGC), where $\left\langle p_{t}^{2}\right\rangle_{1} / F(\xi)$ plays the same role as the saturation momentum scale $Q_{s}^{2}$ in the CGC model $[11,12]$.

\section{Color Suppression Factor $F(\xi)$}

The suppression factor is determined by comparing the charged hadron transverse momentum spectra from $p p$ and $\mathrm{A}+\mathrm{A}$ collisions. To evaluate the initial value of $F(\xi)$ from data for $\mathrm{Au}+\mathrm{Au}$ collisions, a parameterization of $p p$ events at $200 \mathrm{GeV}$ is used to compute the $p_{t}$ distribution [13]

$$
d N_{c} / d p_{t}^{2}=a /\left(p_{0}+p_{t}\right)^{\alpha}
$$

where a is the normalization factor. $p_{0}$ and $\alpha$ are parameters used to fit the data. This parameterization also can be used for nucleus-nucleus collisions to take into account the interactions of the strings [8]

$$
d N_{c} / d p_{t}^{2}=\frac{a^{\prime}}{\left(p_{0} \sqrt{F\left(\xi_{p p}\right) / F\left(\xi_{A A}\right)}+p_{t}\right)^{\alpha}}
$$

In pp collisions $F(\xi)_{p p} \sim 1$ at these energies due to the low overlap probability. $F(\xi)$ is related to $\xi$ by Eq. (2.2). 


\section{Temperature measurement and thermalization}

The connection between $\xi$ and the temperature $T(\xi)$ involves the Schwinger mechanism (SM) for particle production. The Schwinger distribution for massless particles is expressed in terms of $p_{t}^{2}[14]$

$$
d n / d p_{t}^{2} \sim \exp \left(-\pi p_{t}^{2} / x^{2}\right)
$$

where the average value of the string tension is $\left\langle x^{2}\right\rangle$. The tension of the macroscopic cluster fluctuates around its mean value because the chromo-electric field is not constant. The origin of the string fluctuation is related to the stochastic picture of the QCD vacuum. Since the average value of the color field strength must vanish, it cannot be constant but changes randomly from point to point [15]. Such fluctuations lead to a Gaussian distribution of the string tension

$$
\frac{d n}{d p_{t}^{2}} \sim \sqrt{\frac{2}{<x^{2}>}} \int_{0}^{\infty} d x \exp \left(-\frac{x^{2}}{2<x^{2}>}\right) \exp \left(-\pi \frac{p_{t}^{2}}{x^{2}}\right)
$$

which gives rise to thermal distribution [15]

$$
\frac{d n}{d p_{t}^{2}} \sim \exp \left(-p_{t} \sqrt{\frac{2 \pi}{\left\langle x^{2}\right\rangle}}\right),
$$

with $\left\langle x^{2}\right\rangle=\pi\left\langle p_{t}^{2}\right\rangle_{1} / F(\xi)$. The temperature is expressed as [13, 16]

$$
T(\xi)=\sqrt{\frac{\left\langle p_{t}^{2}\right\rangle_{1}}{2 F(\xi)}} .
$$

We will adopt the point of view that the experimentally determined chemical freeze-out temperature is a good measure of the phase transition temperature, $T_{c}$ [4]. The single string average transverse momentum $\left\langle p_{t}^{2}\right\rangle_{1}$ is calculated at $\xi_{c}=1.2$ with the universal chemical freeze-out temperature of $167.7 \pm 2.6 \mathrm{MeV}$ [5]. This gives $\sqrt{\left\langle p_{t}^{2}\right\rangle_{1}}=207.2 \pm 3.3 \mathrm{MeV}$ which is close to $\simeq 200 \mathrm{MeV}$ used previously in the calculation of percolation transition temperature [16].

Recently, it has been suggested that fast thermalization in heavy ion collisions can occur through the existence of an event horizon caused by a rapid deceleration of the colliding nuclei [17]. The thermalization in this case is due the Hawking-Unruh effect [18, 19]. In CSPM the strong color field inside the large cluster produces de-acceleration of the primary $q \bar{q}$ pair which can be seen as a thermal temperature by means of Hawking-Unruh effect. This implies that the radiation temperature is determined by the transverse extension of the color flux tube/cluster in terms of the string tension [20].

$$
T=\sqrt{\frac{\sigma}{2 \pi}}
$$

The temperature obtained using Eq. (4.4) was $\sim 193.6 \mathrm{MeV}$ for Au-Au collisions at $\sqrt{s_{N N}}=200$ $\mathrm{GeV}$ in reasonable agreement with $T_{i}=221 \pm 19^{\text {stat }} \pm 19^{\text {sys }} \mathrm{MeV}$ from the enhanced direct photon experiment measured by the PHENIX Collaboration [21]. For Pb-Pb collisions at $\sqrt{s_{N N}}=2.76 \mathrm{TeV}$ the temperature is $\sim 262.2 \mathrm{MeV}$ for $0-5 \%$ centrality, which is expected to be $\sim 35 \%$ higher than the temperature from $\mathrm{Au}-\mathrm{Au}$ collisions [13]. A recent summary of the results from $\mathrm{Pb}-\mathrm{Pb}$ collisions at the LHC has mentioned that the initial temperature increases at least by $30 \%$ as compared to the top RHIC energy [22]. The direct photon measurements from ALICE gives the temperature of $T_{i}$ $=304 \pm 51 \mathrm{MeV}[23]$. 


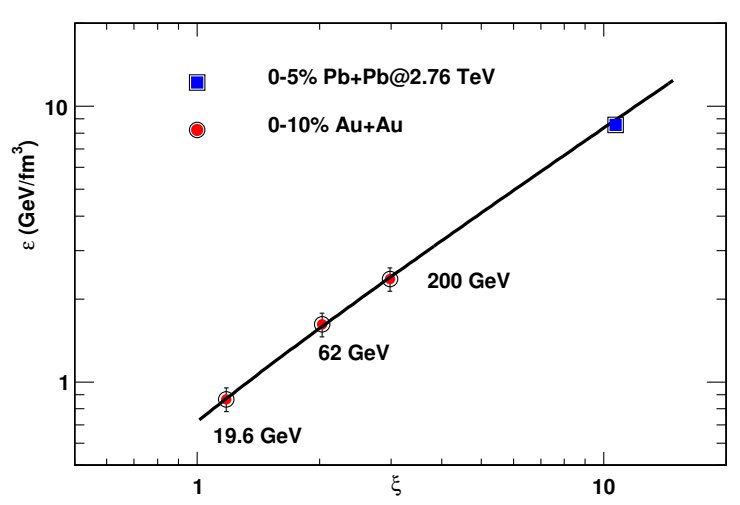

Figure 2: Energy density $\varepsilon$ as a function of the percolation density parameter $\xi$. The extrapolated value for LHC energy is shown as blue square [25].

\section{Energy Density}

Among the most important and fundamental problems in finite-temperature QCD are the calculation of the bulk properties of hot QCD matter and characterization of the nature of the QCD phase transition. The QGP according to CSPM is born in local thermal equilibrium because the temperature is determined at the string level. After the initial temperature $T>T_{c}$ the CSPM perfect fluid may expand according to Bjorken boost invariant 1D hydrodynamics [24]

$$
\varepsilon=\frac{3}{2} \frac{\frac{d N_{c}}{d y}\left\langle m_{t}\right\rangle}{S_{n} \tau_{p r o}}
$$

where $\varepsilon$ is the energy density, $S_{n}$ nuclear overlap area, and $\tau$ the proper time. Above the critical temperature only massless particles are present in CSPM. To evaluate $\varepsilon$ we use the charged pion multiplicity $d N_{c} / d y$ at midrapidity and $S_{n}$ values from STAR for $0-10 \%$ central Au-Au collisions at $\sqrt{s_{N N}}=200 \mathrm{GeV}$ [13]. The factor $3 / 2$ in Eq. (5.1) accounts for the neutral pions. The average transverse mass $\left\langle m_{t}\right\rangle$ is given by $\left\langle m_{t}\right\rangle=\sqrt{\left\langle p_{t}\right\rangle^{2}+m_{0}^{2}}$, where $\left\langle p_{t}\right\rangle$ is the transverse momentum of pion and $m_{0}$ being the mass of pion.

$$
\tau_{\text {pro }}=\frac{2.405 \hbar}{\left\langle m_{t}\right\rangle}
$$

In CSPM the total transverse energy is proportional to $\xi$. From the measured value of $\xi$ and $\varepsilon$, as shown in Fig. 2, it is found that $\varepsilon$ is proportional to $\xi$ for the range $1.2<\xi<2.88, \varepsilon_{i}=0.788 \xi$ $\mathrm{GeV} / \mathrm{fm}^{3}$ [25]. This relationship has been extrapolated to below $\xi=1.2$ and above $\xi=2.88$ for the energy density calculations normalized to $T^{4}$. Figure 3 shows $\varepsilon / T^{4}$ as obtained from CSPM along with the Lattice QCD results from HotQCD Collaboration [26].

\section{Shear Viscosity}

The relativistic kinetic theory relation for the shear viscosity over entropy density ratio, $\eta / s$ is 


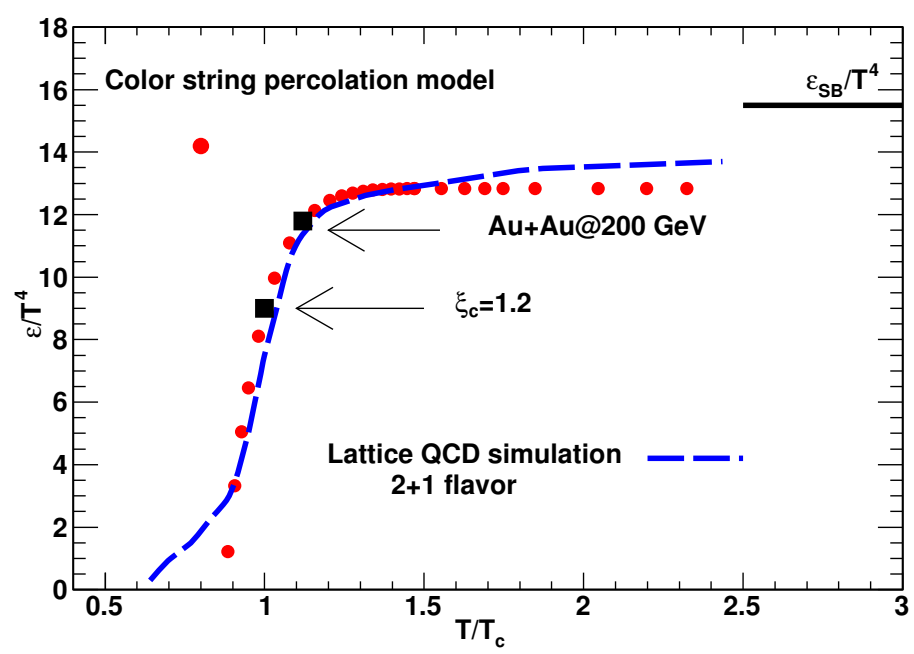

Figure 3: $\varepsilon / T^{4}$ versus $T / T_{c}$ from CSPM (red circles) and Lattice QCD (blue dash line) for 2+1 flavor and p4 action [26].

given by [27]

$$
\frac{\eta}{s} \simeq \frac{T \lambda_{m f p}}{5}
$$

where $\mathrm{T}$ is the temperature and $\lambda_{m f p}$ is the mean free path. $\lambda_{m f p} \sim \frac{1}{\left(n \sigma_{t r}\right)}$ where $n$ is the number density of an ideal gas of quarks and gluons and $\sigma_{t r}$ the transport cross section. In CSPM the number density is given by the effective number of sources per unit volume [25]

$$
n=\frac{N_{\text {sources }}}{S_{N} L}
$$

$\mathrm{L}$ is the longitudinal extension of the source, $\mathrm{L}=1 \mathrm{fm} . \eta / \mathrm{s}$ is obtained from $\xi$ and the temperature

$$
\frac{\eta}{s}=\frac{T L}{5\left(1-e^{-\xi}\right)}
$$

Figure 4 shows a plot of $\eta / s$ as a function of $T / T_{c}$. The lower bound shown in Fig. 4 is given by $\mathrm{AdS} / \mathrm{CFT}$ [29]. The results from $\mathrm{Au}+\mathrm{Au}$ at $200 \mathrm{GeV}$ and $\mathrm{Pb}+\mathrm{Pb}$ at $2.76 \mathrm{TeV}$ collisions show that the $\eta / s$ value is 2.5 and 3.3 times the KSS bound [29].

\section{7. $\eta / s$ to Scaled jet quenching parameter $\hat{q} / T^{3}$}

The small shear viscosity of the QGP implies strong jet quenching. It has been suggested that the jet quenching parameter $\hat{q}$ can also be used to measure the coupling strength of the medium. The shear viscosity $\eta$ of a weakly coupled plasma can be related to the transport parameter for a thermal parton $\hat{q}[30,31]$.

$$
\frac{\eta}{s} \approx \frac{3}{2} \frac{T^{3}}{\hat{q}}
$$

The relation associates a small ratio of $\eta / s$ to a large value of $\hat{q}$. A large amount of theoretical work has been done to extract $\hat{q}$ from jet quenching at RHIC and LHC energies [30, 31, 32, 33]. The 


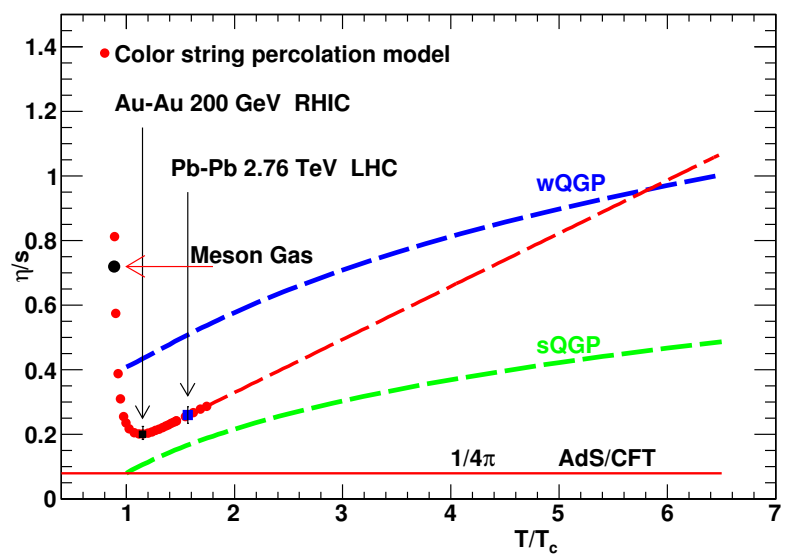

Figure 4: $\eta / s$ as a function of $\mathrm{T} / T_{c}$. Au+Au at $200 \mathrm{GeV}$ for $0-10 \%$ centrality is shown as solid black square. The estimated value for $\mathrm{Pb}+\mathrm{Pb}$ at $2.76 \mathrm{TeV}$ for $0-5 \%$ centrality is shown as a solid blue square. The red dotted line represents the extrapolation to higher temperatures from the CSPM. The hadron gas value for $\eta / s \sim 0.7$ is shown as solid black circle at $\mathrm{T} / T_{c} \sim 0.88[28]$.

latest study by the JET Collaboration has extracted or calculated $\hat{q}$ from five different approaches to the parton energy loss in a dense medium. The evolution of bulk medium in the study was given by $2+1 \mathrm{D}$ or 3+1D hydrodynamic models with the initial temperatures of $T_{R H I C}^{H y d r o}=346-373 \mathrm{MeV}$ and $T_{L H C}^{H y d r o}=447-486 \mathrm{MeV}$ for most central Au+Au collisions at $\sqrt{s_{N N}}=200 \mathrm{GeV}$ and $\mathrm{Pb}+\mathrm{Pb}$ collisions at $\sqrt{s_{N N}}=2.76 \mathrm{TeV}$ respectively. The variation of $\hat{q}$ values between different models can be considered as theoretical uncertainties. One therefore can extract its range of values at RHIC and LHC [32, 33].

$$
\frac{\hat{q}}{T^{3}} \approx\left\{\begin{array}{l}
4.5 \pm 1.3 \text { at } R H I C \\
3.7 \pm 1.4 \text { at } L H C
\end{array},\right.
$$

at the highest temperatures reached in the most central $\mathrm{Au}+\mathrm{Au}$ collisions at $\mathrm{RHIC}$ and $\mathrm{Pb}+\mathrm{Pb}$ collisions at LHC. The corresponding absolute values for $\hat{q}\left(\mathrm{GeV}^{2} / \mathrm{fm}\right)$ for a $10 \mathrm{GeV}$ quark jet are,

$$
\hat{q} \approx \begin{cases}1.2 \pm 0.3 & T=370 M e V \\ 1.9 \pm 0.7 & T=470 M e V\end{cases}
$$

at an initial time $\tau_{0}=0.6 \mathrm{fm} / \mathrm{c}$. The temperature dependence of scaled jet transport parameter $\frac{\hat{q}}{T^{3}}$ is shown in Fig. 5. The CSPM values are shown as solid green squares while the theoretical values are shown as blue squares. It is observed that CSPM values are in agreement with the JET Collaboration results.

\section{8. $\eta / s$ and Trace anomaly $\Delta$}

The trace anomaly $(\Delta)$ is the expectation value of the trace of the energy-momentum tensor, $\left\langle\Theta_{\mu}^{\mu}\right\rangle=(\varepsilon-3 p)$, which measures the deviation from conformal behavior and thus identifies the interaction still present in the medium [34]. We find that the reciprocal of $\eta / s$ is in quantitative agreement with $(\varepsilon-3 p) / T^{4}$ over a wide range of temperatures $[35,36]$. This result is shown in Fig. 6. The minimum in $\eta / s \sim 0.20$ determines the peak of the interaction measure $\sim 5$ in agreement with the recent HotQCD values [37]. This happens at the critical temperature of $T_{c} \sim 175$ 


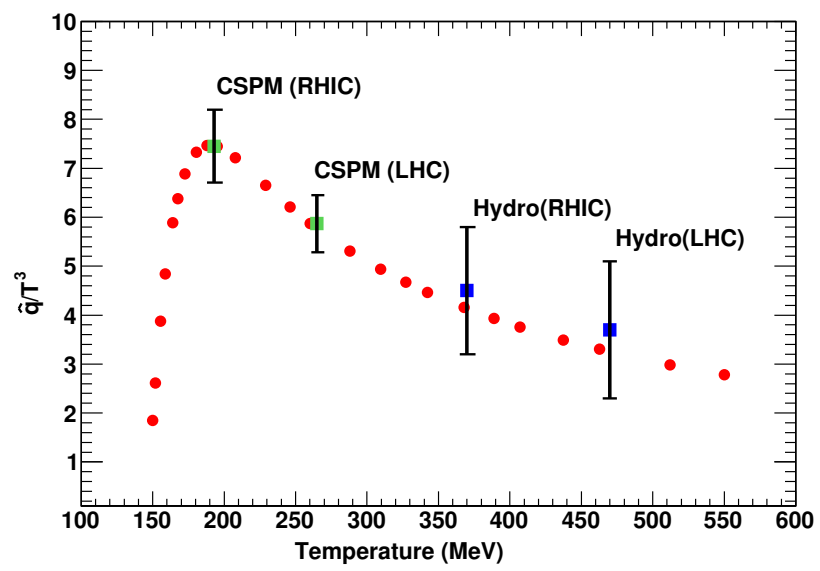

Figure 5: Scaled jet quenching parameter $\frac{\hat{q}}{T^{3}}$ as a function of the temperature. The values shown in solid blue squares Hydro(RHIC) and Hydro(LHC) are given by Eq. (7.2). The CSPM values are shown in green solid squares CSPM(RHIC) and CSPM(LHC) for temperatures $\sim 193$ and $\sim 262 \mathrm{MeV}$ at RHIC and LHC energies, respectively.

MeV. Figure 6 also shows the results from Wuppertal Collaboration [38]. The maximum in $\Delta$ corresponds to the minimum in $\eta / s$. Both $\Delta$ and $\eta / s$ describe the transition from a strongly coupled QGP to a weakly coupled QGP.

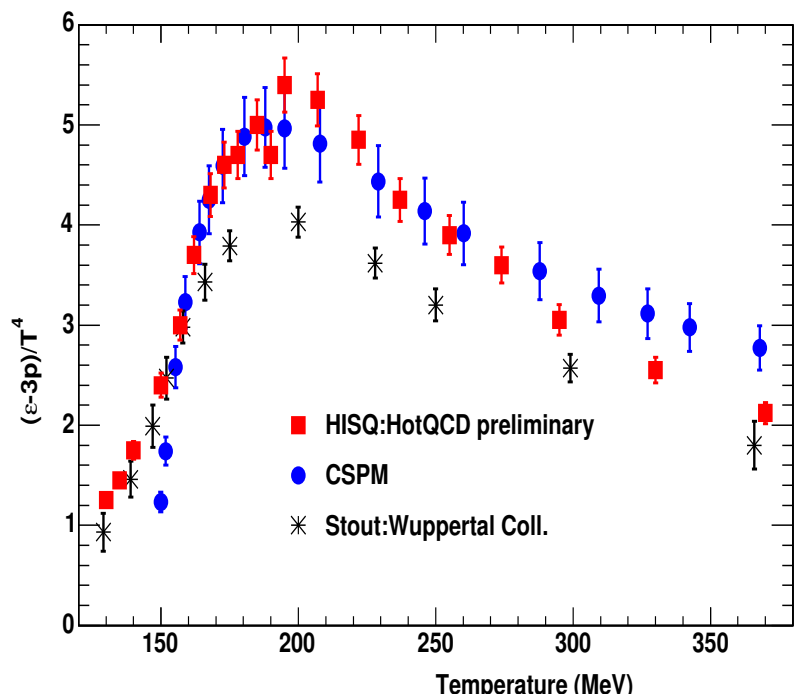

Figure 6: The trace anomaly $\Delta=(\varepsilon-3 p) / T^{4}$ vs temperature [37]. Red solid squares are from HotQCD Collaboration [37]. Black stars are from Wuppertal Collaboration [38].

\section{Equation of State EOS : The sound velocity $C_{s}^{2}$}

An analytic expression for the equation of state, the sound velocity $C_{s}^{2}$ is obtained in CSPM. After the initial temperature $T>T_{c}$ the CSPM perfect fluid may expand according to Bjorken boost invariant $1 \mathrm{D}$ hydrodynamics [24]. The input parameters the initial temperature $\mathrm{T}$, the initial energy 
density $\varepsilon$, and the trace anomaly $\Delta$ are determined by data. The Bjorken 1D expansion gives the sound velocity

$$
\begin{aligned}
\frac{1}{T} \frac{d T}{d \tau} & =-C_{s}^{2} / \tau \\
\frac{d \varepsilon}{d \tau} & =-T s / \tau
\end{aligned}
$$

where $\varepsilon$ is the energy density, s the entropy density, $\tau$ the proper time, and $C_{s}$ the sound velocity. Since $s=\varepsilon+p / T$ and $p=\left(\varepsilon-\Delta T^{4}\right) / 3$ one gets

$$
\frac{d T}{d \varepsilon} s=C_{s}^{2}
$$

From above equations $C_{s}^{2}$ can be expressed in terms of $\xi$

$$
\begin{aligned}
C_{s}^{2} & =(-0.33)\left(\frac{\xi e^{-\xi}}{1-e^{-\xi}}-1\right) \\
& +(\Delta / 3)\left(\frac{0.019}{1-e^{-\xi}}\right)\left(\frac{\xi e^{-\xi}}{1-e^{-\xi}}-1\right)
\end{aligned}
$$

Since there is no direct way to obtain pressure in the CSPM, we have made the assumption that $\Delta=(\varepsilon-3 P) \approx 1 /(\eta / s)$. Figure 7 shows a plot of $C_{s}^{2}$ as a function of $T / T_{c}$. It is observed that the CSPM results are in very good agreement with the lattice calculations [39]. This suggests that the $\Delta$ can be approximated to $1 /(\eta / s)$.

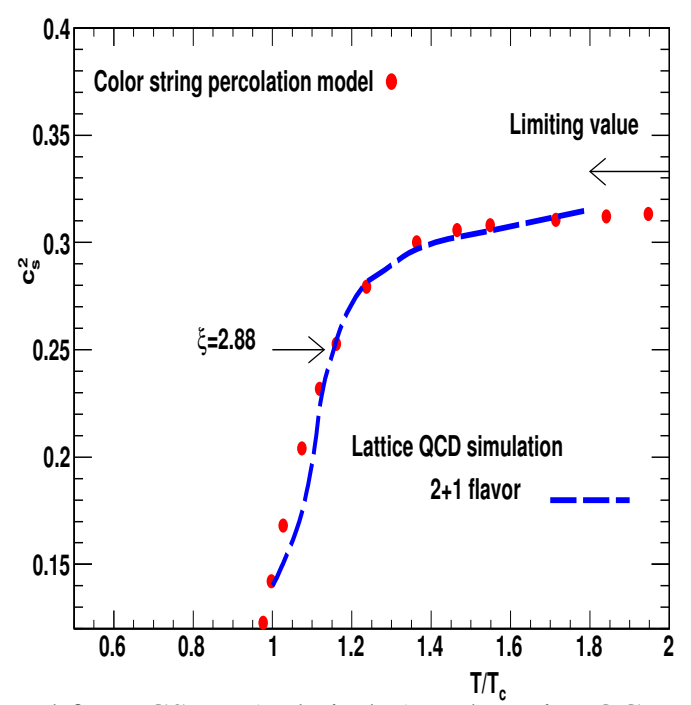

Figure 7: The speed of sound from CSPM (red circles) and Lattice QCD-p4 versus $T / T_{c}$ (blue dash line) [39].

\section{Discussion}

We have shown that the inverse of the shear viscosity to entropy density ratio is able to give good description of the trace anomaly. The jet quenching transport coefficient $\hat{q}$ is also obtained 
using the relation with $\eta / s$. It is observed that scaled jet quenching parameter shows a pronounced maximum close to the critical temperature as seen in the trace anomaly. This indicates that jet quenching parameter can characterize the phase transition [40, 41]. It has been also shown, with in a phenomenological quasi-particle approach, that trace anomaly has a pronounced peak near the critical temperature [42, 43].

The clustering of color sources has shown that the determination of $\eta / s$ as a function of temperature is an important quantity that relates to another transport coefficient, $\hat{q}$ and the trace anomaly $\Delta$. The main assumption of the present approach is that the inverse of $\eta / s$ represents the trace anomaly, $\Delta=(\varepsilon-3 p) / T^{4}$. The clustering of color sources (percolation) provides us with a microscopic partonic picture that connects the transport properties of the QGP to its thermodynamics.

\section{Acknowledgment}

This research was supported by the Office of Nuclear Physics within the U.S. Department of Energy Office of Science under Grant No. DE-FG02-88ER40412. The author thanks A. Hirsch, C. Pajares and R. Scharenberg for fruitful discussions.

\section{References}

[1] T. Celik, F. Karsch, H. Satz, Phys. Lett. B97, 128 (1980).

[2] M. B. Isichenko, Rev. Mod. Phys.64, 961 (1992).

[3] H. Satz, Extreme States of Matter in Strong Interaction Physics, Lecture Notes in Physics 841 ( Springer 2012).

[4] P. Braun-Munzinger, J. Stachel, Christof Wetterich, Phys. Lett. B596, 61 (2004).

[5] F. Becattini, P. Castorina, A. Milov, H. Satz, Eur. Phys. J. C66, 377 (2010).

[6] J. Schwinger, Phys. Rev. 82, 664 (1951).

[7] M. A. Braun, C. Pajares, Eu. Phys. J. C16, 349 (2000).

[8] M. A. Braun, F. del Moral, C. Pajares, Phys. Rev. C 65, 024907 (2002).

[9] M. A. Braun et al., To appear in Phys. Rep. (2015).

[10] H. Satz, Rep. Prog. Phys. 63, 1511 (2000).

[11] L. McLerran, R. Venugopalan, Phys. Rev. D49, 2233 (1994); 3352 (1994).

[12] J. Dias de Deus and C. Pajares, Phys. Lett. B695, 455 (2011).

[13] R. P. Scharenberg, B. K. Srivastava and A. S. Hirsch, Eur. Phys. J. C71, 1510 (2011).

[14] C. Y. Wong, Introduction to high energy heavy ion collisions (World Scientific, 1994).

[15] A. Bialas, Phys. Lett. B466, 301 (1999).

[16] J. Dias de Deus and C. Pajares, Phys. Lett. B642, 455 (2006).

[17] D. Kharzeev and E. Levin and K. Tuchin, Phys. Rev. C75, 044903 (2007).

[18] S. W. Hawking, Commun. Math. Phys. 43,199 (1975). 
[19] W. G. Unruh, Phys. Rev. D14, 870 (1976).

[20] P. Castorina, D. Kharzeev and H. Satz, Eur. Phys. J. C 52, 187 (2007).

[21] A. Adare et al., PHENIX Collaboration, Phys. Rev. Lett. 104, 132301 (2010).

[22] B. Muller, J. Schukraft and W. Wyslouch, Ann. Rev. Nucl. Part. Sci. 62, 361(2012).

[23] K. Safarik, Nucl. Phys. A 904-905, 27c (2013).

[24] J. D. Bjorken, Phys. Rev. D27, 140 (1983).

[25] J. Dias de Deus, A. S. Hirsch, C. Pajares, R. P. Scharenberg, B. K. Srivastava, Eur. Phys. J. C72, 2123 (2012).

[26] A. Bazavov et al., Phys. Rev. D80, 014504 (2009).

[27] T. Hirano and M. Gyulassy, Nucl. Phys. A 769, 71 (2006).

[28] M. Prakash et al., Phys. Rep. 227 , 321 (1993).

[29] P. K. Kovtun, D. T. Son, A. O. Starinets, Phys. Rev. Lett. C 94, 111601 (2005).

[30] A. Majumder, B. Muller and Xin-Nian Wang, Phys. Rev. Lett. 99, 192301 (2007).

[31] Jorge Casalderrey-Solana and Xin-Nian Wang, Phys. Rev. C 77, 024902 (2008).

[32] K. M. Burke et al. (The Jet Collaboration), Phys. Rev. C 90, 014909 (2014).

[33] Xin-Nian Wang, arXiv:1403.3541

[34] M. Cheng et al., Phys. Rev. D 81, 054504 (2010).

[35] R. P. Scharenberg, PoS 017 (CPOD 2013).

[36] B. K. Srivastava, Nucl. Phys. A 926, 142 (2014).

[37] P. Petreczky, Lattice 2012, Cairns, Australia, 2012.

[38] S. Borsanyi et al., Phys. Lett. 730, 99 (2014).

[39] A. Bazavov et al., Phys. Rev. D 80, 014504 (2009).

[40] D. Li, J. Liao and M. Huang, Phys. Rev. D 89, 126006 (2014).

[41] J. Liao and E. Shuryak, Phys. Rev. Lett. 102, 202302 (2009).

[42] M. Bluhm and M. Nahrgang, arXiv:1209.3507.

[43] P. Castorina, D. E. Miller and H. Satz, Eur. Phys. J. C 71, 1673 (2011). 